\title{
Small intestinal length: a factor essential for gut adaptation
}

\author{
L T Weaver, S Austin, T J Cole
}

\begin{abstract}
Changes in small intestinal structure, cytokinetics, and function are dynamic ways in which the gut adapts to diet, disease, and damage. Adequate length provides a static 'reserve' permitting an immediate response to pathophysiological changes. The length of the small intestine from conception to adulthood using data taken from eight published reports of necropsy measurement of 1010 guts is described. Mean length at 20 weeks' gestation was $125 \mathrm{~cm}$, at 30 weeks' $200 \mathrm{~cm}$, at term 275 $\mathrm{cm}$, at 1 year $380 \mathrm{~cm}$, at 5 years $450 \mathrm{~cm}$, at 10 years $500 \mathrm{~cm}$, and at 20 years $575 \mathrm{~cm}$. Prenatal small intestinal growth exceeded that of body length according to the law: small intestinal length $\alpha$ body length to the power 4/3. After birth there was a noticeable deceleration: small intestinal length $\alpha$ body length to the power $1 / 2$. The coefficient of variation of small intestinal length postnatally was $24 \%$, sixfold greater than for body length. The rapid prenatal small intestinal growth rate ensures that the mature newborn has adequate small intestine to meet postnatal nutritional demands, but handicaps the preterm infant who undergoes intestinal resection. The wide variation in lengths suggests a 'surplus' surface area that is immediately available to respond, independent of dynamic mucosal changes, to fluctuations in food availability, local intestinal disease, damage, rapid transit, and resection.
\end{abstract}

The small intestine is the major site of nutrient and water absorption. To ensure an adequate surface area to meet the nutritional requirements of the organism, it is a long coiled organ whose epithelial surface is increased many times by circular folds, villi, and microvilli. ${ }^{\prime}$

For most mammals there is a direct relation between the gross surface area of the small intestine and body size. This is related to the diet eaten by each species, and for man it conforms with that of other members of the superfamily Hominoidea. $^{2}$

The changing rates of small intestinal growth during embryological, prenatal, and postnatal life, and the considerable 'surplus' length of the mature gut have important implications for our understanding and expectations of alimentary function after intestinal resection (particularly in the preterm infant) and during disease, altered food availability, and other pathophysiological conditions.

The purpose of this review is to summarise the published data on the growth of the small intestine of man and to draw attention to two significant findings: (1) the doubling of its length prenatally during the last trimester of pregnancy and (2) the wide variation in the small intestinal lengths of healthy adults.

\section{Subjects and methods}

Reported measurements of small intestinal length were obtained from eight published reports. ${ }^{3-10}$ All data were derived from unfixed necropsy specimens that were usually measured (when methods were described) along the unstretched antimesenteric border from the pylorus or ligament of Treitz to the ileocaecal valve. Subjects with congenital gastrointestinal and cardiac disease have been excluded from the analysis. No distinction has been made between the sexes.

The lengths of 1010 specimens were plotted against the lengths or heights of the subjects. All length measurements were converted to $\mathrm{cm}$. To establish the relation between intestinal length and body length or height, the data were fitted by cubic spline regression." To deal with the mixture of individual and grouped data in the sample, each point in the analysis was given a weighting corresponding to the number of individuals contained in it: 1 for individuals and $n$ for groups of size $n$. The spline curve was fitted to both the original data and to the log transformed data. In the latter case, the slope of the regression curve indicated the power relation between small intestinal length and body length.

\section{Results}

During the first half of pregnancy (to $32 \mathrm{~cm}$ fetal length) there was a linear increase in small intestinal length. Thereafter there was an accelerated rate of growth, with a doubling of small intestinal length during the last 15 weeks of gestation leading to a mean length at birth of $275 \mathrm{~cm}$ (Fig 1).

Growth in intestinal length continued during early postnatal life, but from about 1 year $(75 \mathrm{~cm}$ body length) onwards it slowed and remained linear with increasing age to adulthood. From birth there was a wide range in intestinal lengths reported, with $100 \%$ variation from early childhood onwards. The coefficient of variation of small intestinal length postnatally was $24 \%$, sixfold greater than for body length.

Figure 2 shows the corresponding results and spline curve obtained after log transformation of the data. The pre- and perinatal acceleration in length is clearly seen. In proportional terms, prenatal growth in small intestinal length was consistently faster than that of body length, increasing as the power law small intestinal length $\alpha$ body length to the power $4 / 3$. After birth there was a noticeable deceleration in small intestinal length growth, so that it was less than 


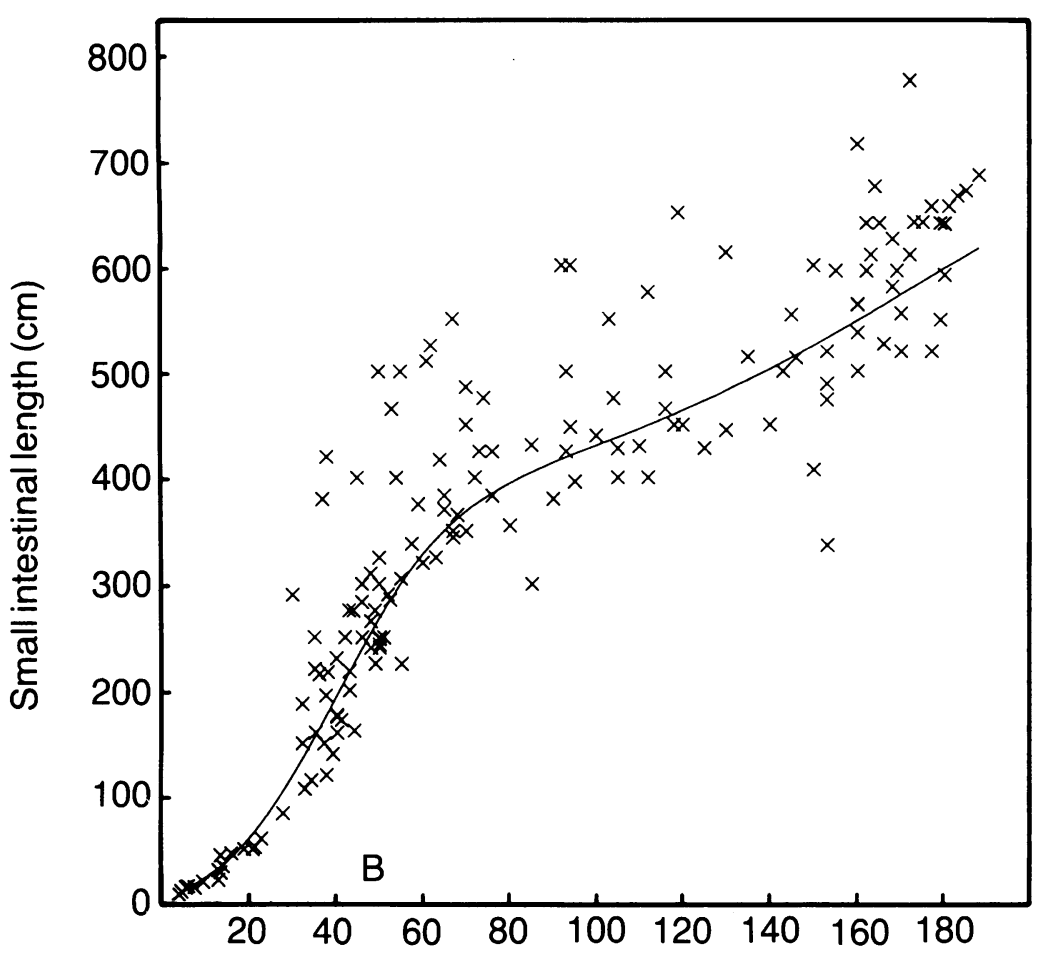

Length of subject $(\mathrm{cm})$

Figure 1: Small intestinal length from early fetal life to maturity. Body length at birth $(B)$ is $50 \mathrm{~cm}$, at one year is $75 \mathrm{~cm}$, at 5 years is $108 \mathrm{~cm}$, at 10 years is $137 \mathrm{~cm}$ and at 20 years is $170 \mathrm{~cm}$. The data were taken from references 3-10 and the smooth curve was fitted by cubic spline regression.

for length according to the relation, small intestinal length $\alpha$ body length to the power $1 / 2$.

\section{Discussion}

Our findings show that the major period of small

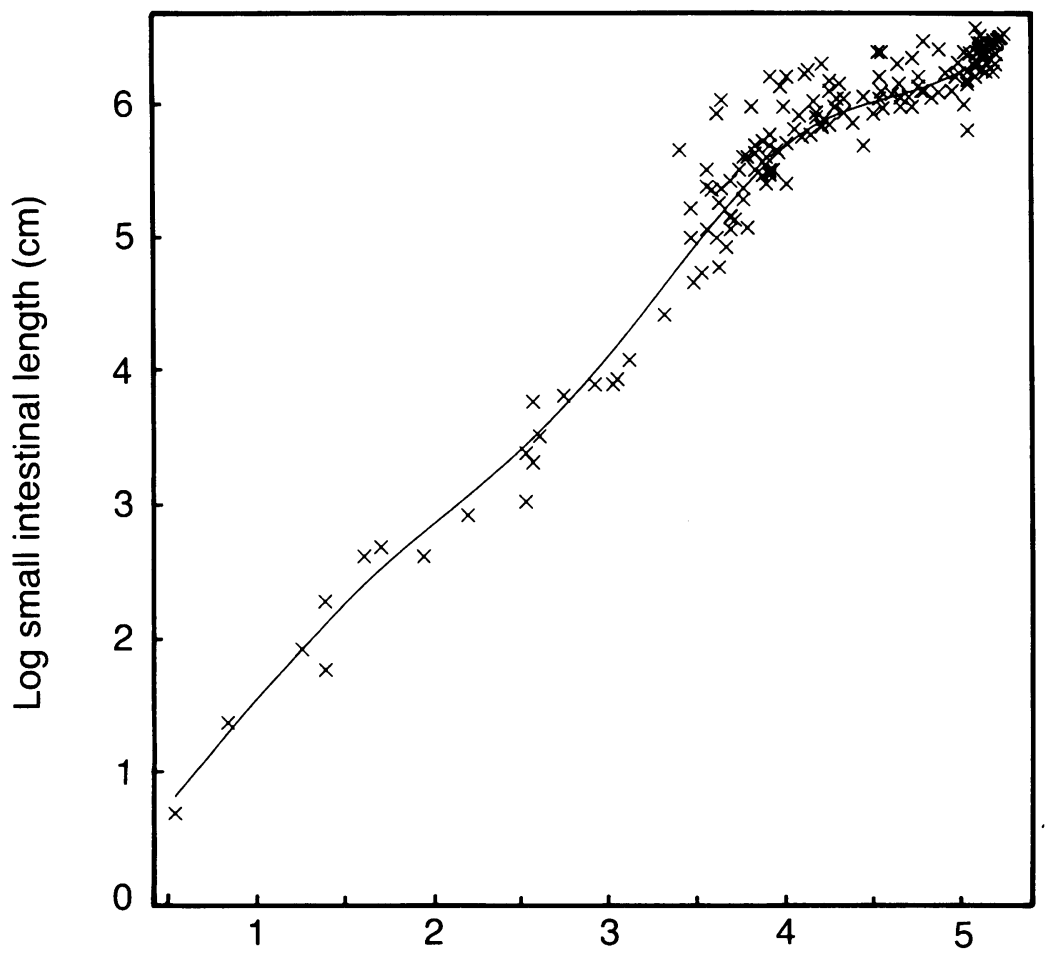

Log subject length $(\mathrm{cm})$

Figure 2: Log transformation of data showing different rates of small intestinal growth pre-, peri-, and postnatally. The data were taken from references 3-10 and the smooth curve was fitted by cubic spline regression. intestinal growth is prenatal, ensuring that the term infant is born with adequate absorptive surface area to support its postnatal nutritional needs. Gastrointestinal development and cellular differentiation occur largely in utero during the first half of pregnancy. During this time there is linear growth of the small intestine, which achieves its final anatomic position in the abdomen, after extracoelomic extension and rotation, by the 20th week of gestation. ' Thereafter there is accelerated growth in length to term. After birth small intestinal growth continues at a slower rate until adulthood.

To establish the relation between intestinal length and body length or height, the cubic spline regression was fitted to the data. ${ }^{.1}$ This is a form of regression which fits a smooth curve to the data without dictating its shape, unlike, for example, polynomial regression. In this way the underlying shape of the relation is emphasised, highlighting in particular the pre- and postnatal growth rates.

The perinatal period is of critical importance to the growing organism, during its adaptation from intrauterine to extrauterine nutrition. Throughout life adaptation seems to be regulated largely by luminal nutrition. Exposure of the neonatal small intestine to enteral feeds is followed by changes in mucosal structure and function: an acceleration of epithelial cell turnover, ${ }^{12}$ activation of absorptive processes, ${ }^{13}$ and a decline in passive permeability. ${ }^{14}$ In the face of starvation or under-nutrition there is atrophy of the mucosa, with shortening of the small intestinal villi, leading to a reduction of absorptive surface area. ${ }^{15}$ Conversely, during pregnancy and lactation, when there is a rise in nutrient requirements, the intestine undergoes hypertrophy, with an increase in length, diameter, villus height, and mucosal surface area. ${ }^{15}{ }^{16}$ Starvation atrophy is not prevented by parenteral nutrition alone, while the hypertrophy of lactation is prevented by restricted food intake. The portion of small intestine that remains after resection has considerable capacity for adaptation. In response to enteral nutrition it may enlarge, not only in length and diameter, but also in the dimensions of its villi and crypts. ${ }^{15} 17$ These adaptations, however, occur against a background of more than adequate gastrointestinal structural and functional reserve in the healthy adult. Throughout postnatal life there is a wide variation in small intestinal length, suggesting more than sufficient, or even surplus, absorptive surface area. The mean length of the small intestine of an adult man is about $550 \mathrm{~cm}$, with a range of $350-700 \mathrm{~cm}$ (Fig 1). This range of length represents a $100 \%$ difference between minimum and maximum values, and probably an equally wide range in small intestinal surface area.

The data presented here were obtained from necropsy specimens. Loss of muscle tone after death leads to apparent lengthening of the small intestine ${ }^{18}$ and traction increases this by up to $4 \% .^{9}$ Although these measures of small intestinal length may differ from those made in the living ${ }^{18}$ the data reported here probably describe the true extent of variation of small intestinal lengths.

Sufficient absorptive surface area to ensure 
adequate nutrient and fluid absorption is essential during gastrointestinal diseases that cause rapid intestinal transit (such as gastroenteritis); in those where there is a generalised or local enteropathy (such as coeliac disease, cow's milk protein intolerance, and Crohn's disease); when a portion of the intestine has been resected or bypassed; when the digestive phases of nutrient assimilation are diminished (such as in pancreatic or hepatic disease, after gastrectomy etc); and in the face of starvation, after severe systemic disease, and other causes of undernutrition.

It therefore seems that there are two major ways in which the small intestine adapts to such pathophysiological changes. The first is passively through its possession of more than adequate surface area. The second is actively by accelerated mucosal proliferation. Surplus small intestinal length is an immediate defence against excessive nutritional demands and gastrointestinal disease, while mucosal proliferation permits a secondary, and more finely responsive adaptation to them.

Figure 1 may be used to predict the length of the small intestine at any particular age, and to calculate what length remains after resection. The neonatal period is that of peak prevalence of diseases that may require intestinal resection, leaving the growing infant at a considerable disadvantage during a critical period of his or her life. Loss of an appreciable portion of the small intestine in the preterm infant will have a major impact on the absorptive capacity of the newborn who has not undergone the late gestational acceleration in intestinal growth. ${ }^{19}$

Like the tissues of the liver and pancreas (other unpaired organs of the body), the absorptive area of mucosa of the small intestine probably exceeds its basal needs, ensuring ample reserve in the face of injury or disease. While its exact dimensions are not critical, its surplus length and well developed regenerative capacity make up for its singularity.

Adaptability to variations in food quality, quantity, and availability has been a major factor accounting for the success and diversity of mammalian species, allowing them to exploit a wide range of nutritional niches. Primates have digestive systems adapted to utilise a varied diet, ${ }^{2}$ and they undergo the major phases of intestinal growth and development before they encounter this. Together, the presence of a 'surplus' or 'reserve' small intestinal surface area, and of an epithelium that is plastic and responsive to environmental changes, arms the organism with a robust and flexible means of responding to the physiological and pathological challenges of eating an omnivorous diet and suffering gastrointestinal disease or damage.

1 Weaver LT. Anatomy and embryology of the gastrointestina tract. In: Walker WA, et al, ed. In: Pediatric gastrointestinal disease. Toronto: Decker, 1990: 195-216.

2 Chivers DJ, Hladik CM. Morphology of the gastrointestinal tract in primates: comparisons with other mammals in tract in primates: comparisons with other

relation to diet. F Morphol $1980 ; 166: 337-86$. human intestine. Am f Med Sci 1924; 167: 499-520.

4 Lacroix B, Kedinger M, Simon-Assmann P, Haffen K. Early organogenesis of human small intestine: scanning electron microscopy and brush border enzymology. Gut 1984; 25 925-30.

5 Reiquam CW, Allen P, Akers DR. Normal and abnorma small bowel lengths. Am $\mathcal{F}$ Dis Child 1965; 109: 447-51.

6 Scammon RE, Kittelson JA. The growth of the gastrointestinal tract of the human fetus. Proc Soc Exp Biol Med 1926; 24: 303-7.

7 Siebert JR. Small-intestine length in infants and children. $\mathrm{Am}$ 7 Dis Child 1980; 134: 593-5.

F Dis Child 1980; 134: $593-5$.
8 Touloukian RJ, Walker Smith J. Normal intestinal length in preterm infants. $\mathcal{F}$ Pediatr Surg 1983; 18: 720-3.

9 Underhill BML. Intestinal length in man. BMF 1955; ii 1243-6.

10 Walker-Smith JA. Massive resection of the small intestine. In: Diseases of the small intestine in childhood. London Butterworths, 1988: 380-9.

11 Silverman BW. Some aspects of the spline smoothing approach to non-parametric regression curve fitting. Fournal of the Royal Statistical Society B 1985; 47: 1-52.

12 Klein RM. Small intestinal cell proliferation during development. In: Lebenthal E, ed. Human gastrointestinal development. New York: Raven Press, 1989: 367-92.

13 Kont. New York: Raven Press, 1989: 367-92.

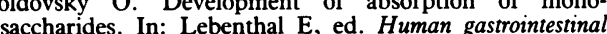
saccharides. In: Lebenthal E, ed. Human gastroi

14 Weaver LT, Laker MF, Nelson R. Intestinal permeability in the newborn. Arch Dis Child 1984; 59: 236-41

15 Dowling RH. Update on intestinal adaptation. Triangle 1988 27: 149-64.

16 Lo CW, Walker WA. Changes in the gastrointestinal tract during enteral and parenteral feeding. Nutr Rev 1989; 47 193-8.

17 Shin CS, Chaudhry AG, Khaddam M, Penha PD, Dooner R. Early morphologic changes in the intestine following massive resection of the small intestine and parenteral nutrition therapy Surg Gynecol Obstetr 1980; 151:246-50.

18 Hirsch J, Ahrens EH, Blankenham DH. Measurement of the human intestinal length in vivo and some causes of variation. haman intestinal length in vivo and

19 Ziegler MM. Short bowel syndrome in infancy: etiology and management. Clin Perinatol 1986; 13: 163-73. 